\title{
MOVING SEMI-INFINITE MODE-III CRACK INSIDE THE SEMI-INFINITE ELASTIC MEDIA
}

\author{
PALAS MANDAL \\ Indian Institute of Technology Hyderabad, Department of Civil Engineering, Telengana, India \\ e-mail: palasmandal146@gmail.com
}

\begin{abstract}
The problem of a semi-infinite moving mode-III crack inside a semi-infinite isotropic half-space is considered. The crack is located between a semi-infinite elastic medium and a layer whose distance from the surface to crack depth is $h$. Initially, Fourier transformation and inverse Fourier transformation are applied to transfer the governing boundary value problem to the well-known Wiener-Hopf equation. The purpose of this problem is to obtain the analytical solution of Stress Intensity Factor (SIF) and Crack Opening Displacement (COD) at the neighbourhood of the crack tip. In numerical Section, I have discussed the variation of parameters (crack velocity, layer distance $h$ from the surface to crack depth) to show the effect of these parametric values on the graphs of SIF and COD where the material properties (density, shear modulus) were fixed.
\end{abstract}

Keywords: semi-infinite crack, isotropic media, Rayleigh wave, SH-wave, stress intensity factor, crack opening displacement

\section{Introduction}

The crack or inclusion related problems with isotropic media has occupied an important place in improving the theory of fracture mechanics. The first and foremost goal of structural engineering is to elude the growth of a crack initiated formerly. A careful undermining into the existing literature reveals that a square root singularity of stress has been found at the crack tip area (Sarkar et al., 1991). In order to predict the state of stress at the vicinity of the crack, we calculate the physical quantity called Stress Intensity Factor. The material properties and applied load are very important parameters for growing the crack. A big amount of researchers solved their problems on fracture mechanics to predict the stress intensity factor. Maximum problems have been solved analytically by researchers but there was arisen some mathematical complication to complete the problems. In that case, numerical approximation is the best way. The difficulty of the problem became more for semi-infinite moving cracks. The moving crack concept was first introduced by Yoffe (1951). He investigated crack propagation with a constant speed without changing in length along the crack propagation axis. A semi-infinite crack was appearing and growing suddenly with a constant speed in a stretched elastic body. The Weiner-Hopf and Cagniard techniques were applied to solve this mixed boundary value problem in the semi-infinite crack. Finally, they calculated the stress value at the vicinity of the crack tip for an asymptotic case. Atkinson and Popelar (1979) discussed spatially changing elastic properties in elastic media with stable state semi-infinite crack propagation. Sih (1968) deliberated the problem of elastodynamics in moving and stationary cracks as well as delivered a formulation of Riemann-Hilbert with consideration of in-plane extensional and anti-plane shear loads. The also provided solution for a constant speed of crack propagation. Knauss (1966) suggested a semi-infinite straight crack which was containing with an infinitely long isotropic strip of finite width by considering a displaced clamped boundary on normal to the crack also discussed asymptotic 
expansion stresses in a sudden crack. After Rice (1967) corrected that work. A simple method was introduced by Nilsson $(1972,1973)$ to handle a semi-infinite moving crack with an isotropic strip. The also used to calculate the stress intensity factor by using Wiener-Hopf technique. Lowengrub (1975) and Atkinson and Popelar (1979) considered for the solution of a couple of coplanar cracks situated between two glued different isotropic elastic half-planes. To reduce the problem regarding the concurrent pair of triple integral equations with trigonometric kernel, Fourier transformation was applied. Georgiadis (1986) analysed a cracked plane orthotropic strip to give a steady state solution by using the Wiener-Hopf technique. Kuo and Chen (1992) discussed stable three-dimensional stress intensity factors regarding semi-infinite plane cracks with both symmetric as well as anti-symmetric cases. Das and Ghosh (1992) obtained transformation of a pair of Griffith cracks affecting the interface of the pair of a different elastic medium with the limitation of constant velocity under anti-plane shear stress.

In our problems, we considered a semi-infinite moving crack situated at the interface of a layer of some thickness and a semi-infinite half space but of the same material. We finally solved the problem numerically. Li (2001) discussed the explanation for the stress intensity factor of mode III for different elastic layers of the same thickness. The problem has been derived analytically for stress free and clamped boundaries. Finally, the Wiener-Hopf technique has been applied to find analytical solution for a semi-infinite interface crack in two bonded dissimilar layers. Wang et al. (2001) proposed in orthotropic materials with concentrated shear impact, a solution for variable stress intensity factor for a semi-infinite crack. To solve the equation of motion, the Laplace and Fourier transforms together along with the Wiener-Hopf technique were employed. The results are shown for both orthotropic and isotropic materials. Wu et al. (2002) observed a semi-infinite crack between a pair of bonded different elastic strips. Investigation of the analytical solution for different moving cracks of a piezoelectric strip as well as the layers was suggested by Bagheri et al. (2016) and Nourazar and Ayatollahi (2016). The some problems were regarded with the shear wave.

A huge amount of works has been done on this topic by the greatest researchers. The main focus on this topic is the crack (static, moving) and media (isotropic, anisotropic). Most of the problems are solved for anisotropic and isotropic media by static cracks. The number is very small in the case of semi-infinite moving cracks in isotropic media. In my problem, I consider a moving semi-infinite mode III crack at a depth $h$ from the surface in an isotropic elastic medium. The Wiener-Hopf technique is the best method to handle semi-infinite moving crack related problems. Firstly, the Fourier transform is used to convert the boundary value problem to the Wiener-Hopf equation. To find explicit expressions for SIF and COD in the asymptotic cases the Wiener-Hopf technique was applied. Further, I observe the effects of material constants on SIF and COD by plotting different graphs among them.

\section{Description of the problem}

Consider a semi-infinite crack which is moving and parallel to the surface of a semi-infinite elastic medium at a depth $h$ from the surface. The symmetrical cartesian axes of the isotropic materials are $X, Y, Z$. The crack position is $-\infty<X<0, Y=0,-\infty<Z<\infty$. We are assuming that the Rayleigh surface waves velocity is greater than the crack speed $c$.

The displacement component $W(X, Y, t)$ has satisfied the following elastic wave equation

$$
\frac{\partial^{2} W}{\partial X^{2}}+\frac{\partial^{2} W}{\partial Y^{2}}=\frac{1}{c_{s}^{2}} \frac{\partial^{2} W}{\partial t^{2}}
$$




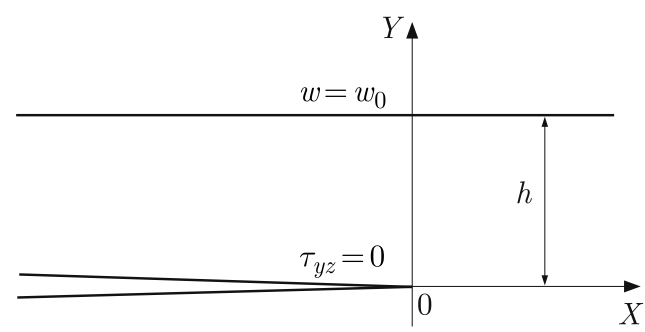

Fig. 1. Geometry of the original problem

where shear wave velocity, density and shear modulus of the materials are respectively $c_{s}=\sqrt{\mu_{12} / \rho}, \rho$ and $\mu_{12}$. We can write $x=X-c t, y=Y, z=Z, t=t$ by using the Galilean transformation, above equation (2.1) becomes

$$
s^{2} \frac{\partial^{2} w}{\partial x^{2}}+\frac{\partial^{2} w}{\partial y^{2}}=0
$$

where $s^{2}=1-c^{2} / c_{s}^{2}, c$ is the crack velocity and $w(x, y)=W(X, Y, t)$.

The relation between the stress and displacement component for othotropic materials is as follows

$$
\tau_{y z}=\mu_{12} \frac{\partial w}{\partial y}
$$

The boundary conditions inside and outside the crack are given by

$$
\begin{array}{ll}
\tau_{y z}(x, 0)=0 & x<0 \\
w(x, 0)=0 & x>0 \\
\tau_{y z}(x, h)=0 & -\infty<x<\infty \\
w(x, h)=w_{0} & -\infty<x<\infty
\end{array}
$$

where $w_{0}$ is fixed.

The Wiener-Hopf technique is not applicable to these types of above boundary conditions. For this reason, I superimpose a constant normal load $\tau_{0}$ on the above system of the boundary conditions to make them appropriate for the numerical method. The new boundary conditions are

$$
\begin{array}{ll}
\tau_{y z}(x, 0)=\tau_{0} & x<0 \\
w(x, 0)=0 & x>0 \\
\tau_{y z}(x, h)=0 & -\infty<x<\infty \\
w(x, h)=0 & -\infty<x<\infty
\end{array}
$$

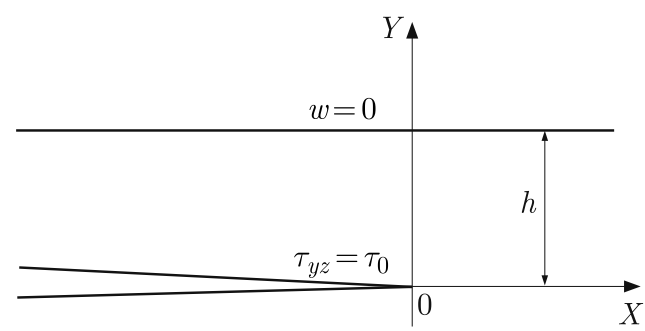

Fig. 2. Geometry of the transformed problem 
Then, by a trivial superimposition we may arrive again at the original problem. Obviously, such a superposition does not affect the value of the stress intensity factor obtained by solving the problem. The above two problems are identical for a particular value of $\tau_{0}$. For generalized plane stress problems the appropriate value of $\tau_{0}$ is given by $-\mu_{12} w_{0} / h$. The complete procedure to find the value of $\tau_{0}$ was elaborated by Georgiadis and Papadopoulos (1988).

Now we can use the following relation in lieu of the constant load $\tau_{0}$ as discussed by Nilsson $(1972,1973)$

$$
\tau_{y z}(x, 0)=\tau_{0} \mathrm{e}^{\varepsilon x}
$$

where $\varepsilon>0$, which is a very slow tending to zero.

The Fourier transform is given by

$$
\bar{f}(\omega, y)=\frac{1}{\sqrt{2 \pi}} \int_{-\infty}^{\infty} f(x, y) \mathrm{e}^{\mathrm{i} \omega x} d x
$$

after inverse

$$
f(x, y)=\frac{1}{\sqrt{2 \pi}} \int_{-\infty}^{\infty} \bar{f}(\omega, y) \mathrm{e}^{-\mathrm{i} \omega x} d \omega
$$

where $\omega=\sigma+\mathrm{i} \zeta, \sigma$ and $\zeta$ are the real and imaginary parts.

After taking the Fourier transform, boundary conditions $(2.5)_{3}$ and $(2.5)_{4}$ become

$$
\bar{w}(\omega, h)=0 \quad \bar{\tau}_{y z}(\omega, 0)=0
$$

Taking Fourier transform on equation (2.2), the solution can be expressed as

$$
\bar{w}(\omega, y)= \begin{cases}A(\omega) \mathrm{e}^{-s \omega y}+B(\omega) \mathrm{e}^{s \omega y} & \text { for } 0 \leqslant y \leqslant h \\ C(\omega) \mathrm{e}^{s \omega y} & \text { for }-\infty<y \leqslant 0\end{cases}
$$

where $\bar{w}(\omega, y)$ be the Fourier transform of the displacement component $w(x, y)$ and $A(\omega)$, where $B(\omega)$ and $C(\omega)$ are unknown functions of the transformed variable $\omega$. The stress components are

$$
\bar{\tau}_{y z}(\omega, y)= \begin{cases}\mu_{12} s \omega\left[B(\omega) \mathrm{e}^{s \omega y}-A(\omega) \mathrm{e}^{-s \omega y}\right] & \text { for } \quad 0 \leqslant y \leqslant h \\ C(\omega) s \omega \mathrm{e}^{s \omega y} & \text { for } \quad-\infty<y \leqslant 0\end{cases}
$$

\section{Procedure of solution}

Let us define two unknown functions to determine the complete solution

$$
\begin{array}{ll}
\tau_{y z}(x, 0)=p(x) & x>0 \\
w(x, 0)=q(x) & x<0
\end{array}
$$

where the shear stress function is $p(x)$ outside the crack along the $x$-axis, and the jump discontinuity displacement function $q(x)$ inside the crack along the $y$-axis. By taking both sides, the Fourier transformation of Eqs. (3.1) is

$$
\bar{p}_{+}(\omega)=\frac{1}{\sqrt{2 \pi}} \int_{0}^{\infty} p(x) \mathrm{e}^{\mathrm{i} \omega x} d x \quad \bar{q}_{-}(\omega)=\frac{1}{\sqrt{2 \pi}} \int_{-\infty}^{0} q(x) \mathrm{e}^{\mathrm{i} \omega x} d x
$$


The functions $p(x)$ and $q(x)$ are bounded at the infinity as the stress and displacements are tending to zero at infinity. The following functions are

$$
\begin{array}{lll}
|p(x)|<P x^{-l_{p}} & \text { as } & x \rightarrow \infty \\
|q(x)|<Q|x|^{-l_{q}} & \text { as } & x \rightarrow-\infty
\end{array}
$$

where $l_{p}>0, l_{q}>0, P>0$ and $Q>0$. Here $P$ and $Q$ are finite. Also it can be shown that the functions $\bar{p}_{+}(\omega)$ and $\bar{q}_{-}(\omega)$ are analytic for $\zeta \geqslant 0$ and $\zeta \leqslant 0$, respectively.

Using boundary condition $(2.9)_{1}$, i.e, $\bar{w}(\omega, h)=0$ we get

$$
B(\omega)=-A(\omega) \mathrm{e}^{-2 s \omega h}
$$

By utilizing boundary condition (2.6), (2.5) 2 with using equations (3.2), we get

$$
\bar{\tau}_{y z}(\omega, 0)=\bar{p}_{+}(\omega)+\frac{\tau_{0}}{\sqrt{2 \pi}(\varepsilon+\mathrm{i} \omega)} \quad \bar{w}(\omega, 0)=\bar{q}_{-}(\omega)
$$

Equation $(3.5)_{1}$ can be rearranged as

$$
\bar{p}_{+}(\omega)=\bar{\tau}_{y z}(\omega, 0)-\frac{\tau_{0}}{\sqrt{2 \pi}(\varepsilon+\mathrm{i} \omega)}
$$

at $y=0$, the solutions are

$$
\bar{w}(\omega, 0)=A(\omega)+B(\omega)
$$

and

$$
\bar{w}(\omega, 0)=C(\omega)
$$

using (3.4) and (3.5) $)_{2}$ in (3.7), we get

$$
A(\omega)=\frac{\bar{q}_{-}(\omega)}{1-\mathrm{e}^{-2 s \omega h}}
$$

From equations $(3.5)_{2}$ and (3.8), we obtain

$$
C(\omega)=\bar{q}_{-}(\omega)
$$

Now equation (3.6) becomes

$$
\bar{p}_{+}(\omega)=L(\omega) \bar{q}_{-}(\omega)-\frac{\tau_{0}}{\sqrt{2 \pi}(\varepsilon+\mathrm{i} \omega)}
$$

where

$$
L(\omega)=\frac{-\mu_{12} s \omega\left(1+\mathrm{e}^{-2 s \omega h}\right)}{1-\mathrm{e}^{-2 s \omega h}}
$$

Equation (3.11) is the well-known Wiener-Hopf equation of the unknown functions $\bar{p}_{+}(\omega)$ and $\bar{q}_{-}(\omega)$. In order to solve the equation, it is essential to factorize the kernel $L(\omega)$ in the following form

$$
L(\omega)=L_{+}(\omega) L_{-}(\omega)
$$

where the functions $L_{+}(\omega)$ and $L_{-}(\omega)$ both are analytic and non-zero for $\zeta>\zeta_{1}\left(\zeta_{1}<0\right)$ and $\zeta<\zeta_{2}\left(\zeta_{2}>0\right)$. Utilizing Eq. (3.12) in Eq. (3.11), we get

$$
\frac{\bar{p}_{+}(\omega)}{L_{+}(\omega)}=L_{-}(\omega) \bar{q}_{-}(\omega)-\frac{\tau_{0}}{\sqrt{2 \pi}(\varepsilon+\mathrm{i} \omega) L_{+}(\omega)}
$$


Now

$$
\frac{\tau_{0}}{\sqrt{2 \pi}(\varepsilon+\mathrm{i} \omega) L_{+}(\omega)}=R(\omega)=R_{+}(\omega)+R_{-}(\omega)
$$

where

$$
R_{+}(\omega)=\frac{\tau_{0}}{\sqrt{2 \pi}(\varepsilon+\mathrm{i} \omega)}\left(\frac{1}{L_{+}(\omega)}-\frac{1}{L_{+}(\mathrm{i} \varepsilon)}\right) \quad R_{-}(\omega)=\frac{\tau_{0}}{\sqrt{2 \pi}(\varepsilon+\mathrm{i} \omega) L_{+}(\mathrm{i} \varepsilon)}
$$

We have checked analyticity of the functions $R_{+}(\omega)$ and $R_{-}(\omega)$ as it was done by Noble (1958). These two functions are non-zero in $\zeta>\zeta_{1}$ and $\zeta<\varepsilon$, respectively. From (3.13) and (3.14), we get

$$
\frac{\bar{p}_{+}(\omega)}{L_{+}(\omega)}+R_{+}(\omega)=L_{-}(\omega) \bar{q}_{-}(\omega)-R_{-}(\omega)
$$

As mentioned earlier, see Eq. (3.11), the regions of analyticity of the functions $\bar{p}_{+}(\omega), \bar{q}_{-}(\omega)$, $L_{+}(\omega), L_{-}(\omega), R_{+}(\omega)$ and $R_{-}(\omega)$ are $\zeta \geqslant 0, \zeta \leqslant 0, \zeta>\zeta_{1}\left(\zeta_{1}<0\right), \zeta<\zeta_{2}\left(\zeta_{2}>0\right)$, $\zeta>\zeta_{1}\left(\zeta_{1}<0\right)$ and $\zeta<\varepsilon$, respectively. The left and the right hand side of equation $(3.16)$ are both analytic at the region of the upper half plane $\zeta \geqslant 0$ and the lower half plane $\zeta \leqslant$ where $\varepsilon>0$. The analytic regions are overlapped. The common part of analyticity is the line $\zeta=0$. Equation (3.16) is totally analytic and single valued in the whole complex plane $\omega$ by analytic continuation. For this reason, we have considered boundary condition (2.6) in lieu of original boundary condition $(2.5)_{1}$ apart from that there is no common region for showing analyticity.

By considering the large values of $\omega$, the functions $L_{+}(\omega)$ and $L_{-}(\omega)$ tend to be $\omega^{1 / 2}$, and the functions $\bar{p}_{+}(\omega)$ and $\bar{q}_{-}(\omega)$ will be bounded. Now we consider Eq. (3.16) equal to $I(\omega)$. This function is analytic as Eq. (3.16) is. The function $I(\omega)$ is tending to $\omega^{-1 / 2}$ for large values of $\omega$ in the upper half plane $\zeta \geqslant 0$. Moreover, using the same arguments the right hand side of equation (3.16) is analytic and tending to $\omega^{1 / 2}$ for large values of $\omega$ in the lower half plane $\zeta \leqslant 0$. It can be culminated that the function $I(\omega)$ is identically zero by the extended Liouville theorem

$$
I(\omega)=0
$$

Now from (3.15), (3.16) and (3.17) we get

$$
\bar{p}_{+}(\omega)=\frac{\tau_{0}}{\sqrt{2 \pi}(\varepsilon+\mathrm{i} \omega)\left(\frac{L_{+}(\omega)}{L_{+}(\varepsilon)}-1\right)} \quad \bar{q}_{-}(\omega)=\frac{\tau_{0}}{\sqrt{2 \pi}(\varepsilon+\mathrm{i} \omega) L_{+}(\mathrm{i} \varepsilon) L_{-}(\omega)}
$$

We can take $\varepsilon \rightarrow 0$ for constant loading and, consequently, the above equations become

$$
\bar{p}_{+}(\omega)=\frac{\tau_{0}}{\sqrt{2 \pi} \mathrm{i} \omega\left(\frac{L_{+}(\omega)}{L_{+}(0)}-1\right)} \quad \bar{q}_{-}(\omega)=\frac{\tau_{0}}{\sqrt{2 \pi} \mathrm{i} \omega L_{+}(0) L_{-}(\omega)}
$$

In the Wiener-Hopf technique, the primary difficulty is to decompose the kernel $L(\omega)$ in Eq. (3.12). Both for very large and small values of $\omega$, Nilsson (1972) gave a report that Stress Intensity Factor (SIF) can be obtained by only knowing the values of $L(\omega)$.

After some lengthy algebraic manipulations, it can be verified that

$$
\lim _{\omega \rightarrow \infty} \frac{L(\omega)}{\omega}=-\mu_{12} s
$$

and

$$
\lim _{\omega \rightarrow 0} L(\omega)=-\frac{2 \mu_{12} s^{2} h+\mu_{12} s}{2 s h}
$$


Equations (3.19) can be written for large values of $\omega$

$$
\begin{aligned}
& \lim _{\omega \rightarrow \infty} \bar{p}_{+}(\omega)=\lim _{\omega \rightarrow \infty} \frac{\tau_{0}}{\sqrt{2 \pi} \mathrm{i}} \frac{L_{+}(\omega)}{\omega^{\frac{1}{2}} \omega^{\frac{1}{2}} L_{+}(0)}-\lim _{\omega \rightarrow \infty} \frac{\tau_{0}}{\sqrt{2 \pi} \mathrm{i} \omega} \\
& \lim _{\omega \rightarrow \infty} \bar{q}_{-}(\omega)=\lim _{\omega \rightarrow \infty} \frac{\tau_{0}}{\sqrt{2 \pi} \mathrm{i} L_{+}(\omega) \omega^{\frac{3}{2}}} \frac{\omega^{\frac{1}{2}}}{L_{-}(\omega)}
\end{aligned}
$$

By using equations (3.20) and (3.21), we can take the inverse Fourier transform on (3.22)

$$
\lim _{x \rightarrow 0^{+}} p(x)=-\tau_{0} \sqrt{\frac{h s}{\pi(2 s h+1)}} x^{-\frac{1}{2}} \quad \lim _{x \rightarrow 0^{-}} q(x)=-\tau_{0} \sqrt{\frac{h}{\pi \mu_{12}^{2}(2 s h+1) s}} x^{\frac{1}{2}}
$$

From the definition of the function $p(x)$, Eq. $(3.23)_{1}$ represents the shear stress component $\tau_{y z}$ along the $x$-axis just outside the crack tip. We got a square root singularity of the shear stress component at the tip of the crack as expected. This result is very expected in the field of Fracture Mechanics. Finally, equation $(3.23)_{2}$ represents the displacement $w$ just inside the crack tip. This quantity known as Crack Opening Displacement (COD) is also important in view of the physical nature of the crack.

\section{SIF and COD}

The state of stress at the crack tip is determined by a quantity called the Stress Intensity Factor (SIF) which is defined by

$$
\mathrm{SIF}=\lim _{x \rightarrow 0^{+}} \sqrt{2 \pi x} \tau_{y z}(x, 0)
$$

obtained as

$$
\mathrm{SIF}=-\tau_{0} \sqrt{\frac{2 h s}{2 s h+1}}
$$

The original Stress Intensity Factor is given by

$$
\mathrm{SIF}=-\mu_{12} w_{0} \sqrt{\frac{2 s}{h(2 s h+1)}}
$$

The other physical quantity of interest is the Crack Opening Displacement (COD)

$$
\mathrm{COD}=w\left(x, 0^{+}\right)-w\left(x, 0^{-}\right)
$$

and

$$
\mathrm{COD}=2 \sqrt{\frac{h}{\pi \mu_{12}^{2} s(2 s h-1)}} x^{\frac{1}{2}}
$$

\section{Results and discussion}

Finally, the expressions (in Eqs. (4.3) and (4.5)) of SIF and COD are obtained by applying the Fourier transformation and Weiner-Hopf technique. From these results, I can confirm that the material properties are very important to find the values of these quantities. The SIF at the 
Table 1

\begin{tabular}{|c|c|c|c|}
\hline & & $\rho_{1}\left[\mathrm{gm} / \mathrm{cm}^{3}\right]$ & $\mu_{1}\left[\mathrm{dyne} / \mathrm{cm}^{2}\right]$ \\
\hline \hline Type-1 & Copper & 8.96 & $4.5 \cdot 10^{11}$ \\
\hline Type-2 & steel & 7.6 & $7.617 \cdot 10^{11}$ \\
\hline Type-3 & aluminium & 2.7 & $2.593 \cdot 10^{11}$ \\
\hline
\end{tabular}

tip of the crack and COD have been plotted graphically against the crack velocity $c$ and the distance $x$ to show the effects of the materials isotropy on the SIF and COD.

In the following table, we provide the well-known isotropic material constants which will be used in appropriate junctures. The material constants of isotropic materials have been taken from Srivastava et al. (1980), see Table 1.

The crack propagation velocity is less than the Rayleigh surface wave velocity in practice. The Rayleigh surface wave velocity is $0.78 \mathrm{~cm} / \mu$ s for Type- 1 material. Figure 3 shows that the stress intensity factor depends on the crack propagation velocity $c$. For plane stress problems, it is clear that the graph of SIF decreases with the increasing value of $c$ and tending to zero as $c$ approaches the Rayleigh surface wave velocity where the crack depth $h$ from the surface is fixed. If $c$ is the Rayleigh surface wave velocity then the SIF will be vanished. In this case, we are taking different basic equations as the graph is no longer valid for super Rayleigh wave velocities.

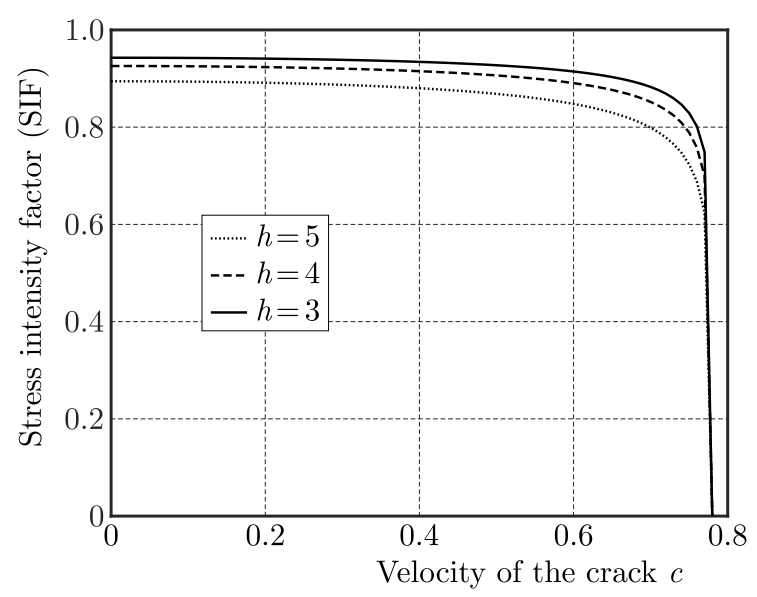

Fig. 3. SIF against the crack velocity $c$ for Type-1 material

Also for a fixed crack propagation velocity $c$, the value of SIF decreases as the crack depth $h$ from the surface increases. The velocities of the Rayleigh waves are $0.881 \mathrm{~cm} / \mu \mathrm{s}$ and $0.982 \mathrm{~cm} / \mu \mathrm{s}$, respectively for Type-2 and Type-3 materials, and the graphs (Fig. 4) are absolutely the same with Fig. 3.

From the COD graphs, it is clearly illustrated that the experimental value of reductions of COD for non-variable values of $h$ as $x$ moves towards the origin and finally tends to zero at the origin. With the physical nature of the crack planes this result is consistent and becoming nearer by nearer as we transfer negative $x$-axis toward the crack tip. Also it is detected that height of the graph falls when the crack depth $h$ increases. This prediction is obtained from Eq. (4.5). The effect of the material constants on the COD is also noticeable as it can be seen that the values of the COD change if the materials are interchanged. 
(a)

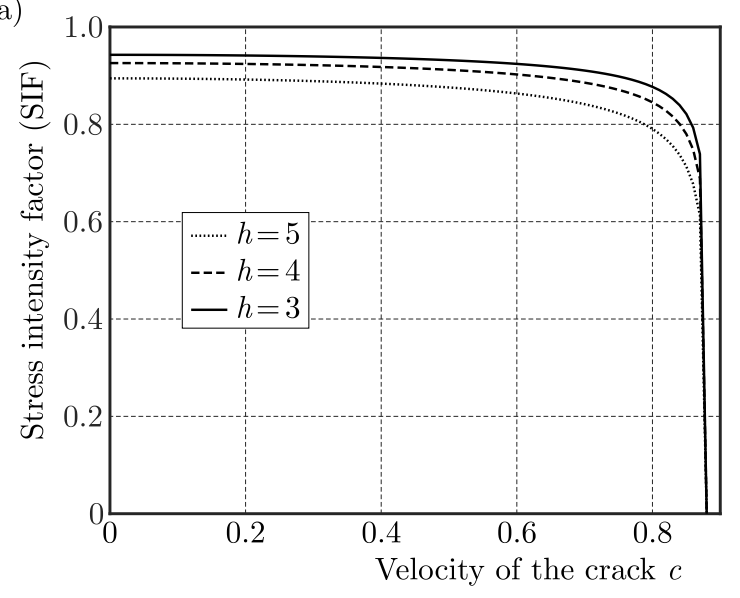

(b)

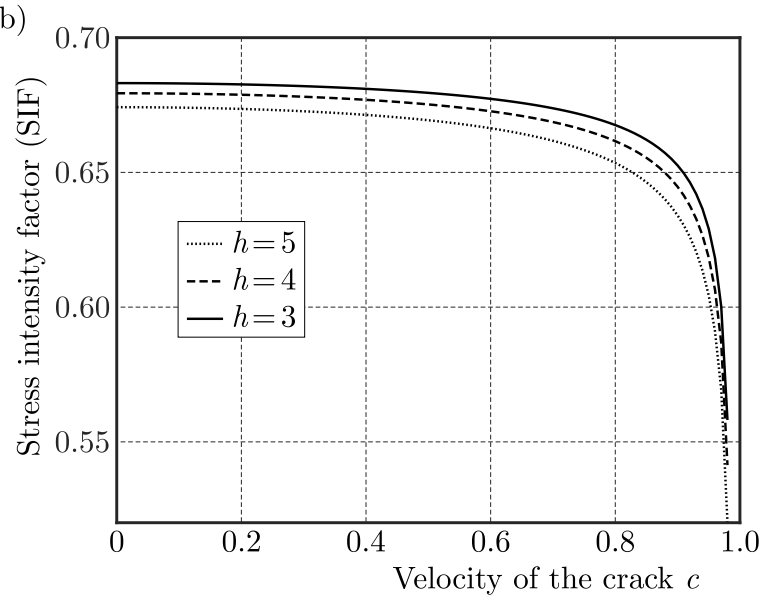

Fig. 4. SIF against crack velocity $c$ for (a) Type-2 and (b) Type-3 material
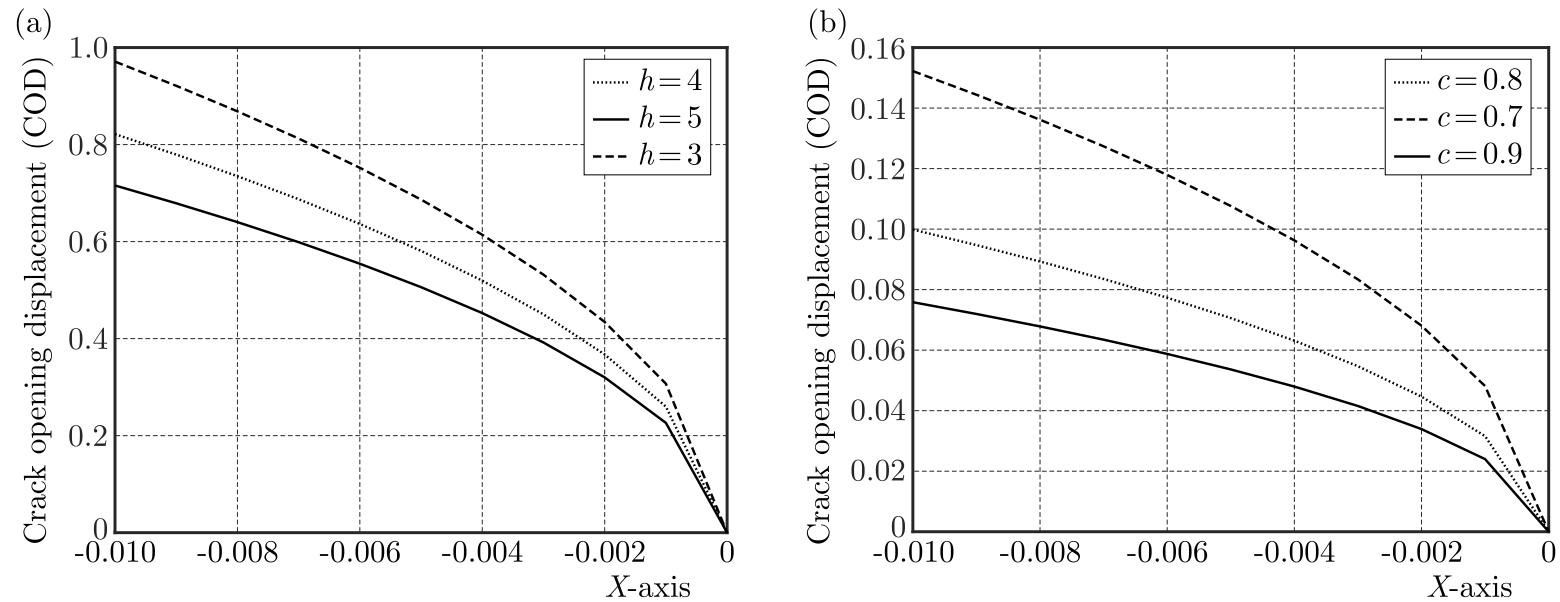

Fig. 5. COD vs. distance $x$ for Type- 1 material, (a) $c=0.2$ and (b) $h=4$

(a)

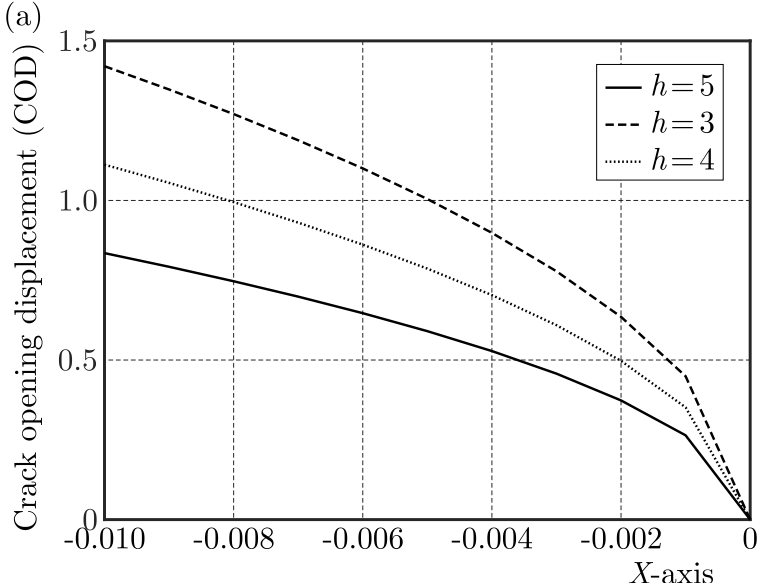

(b)

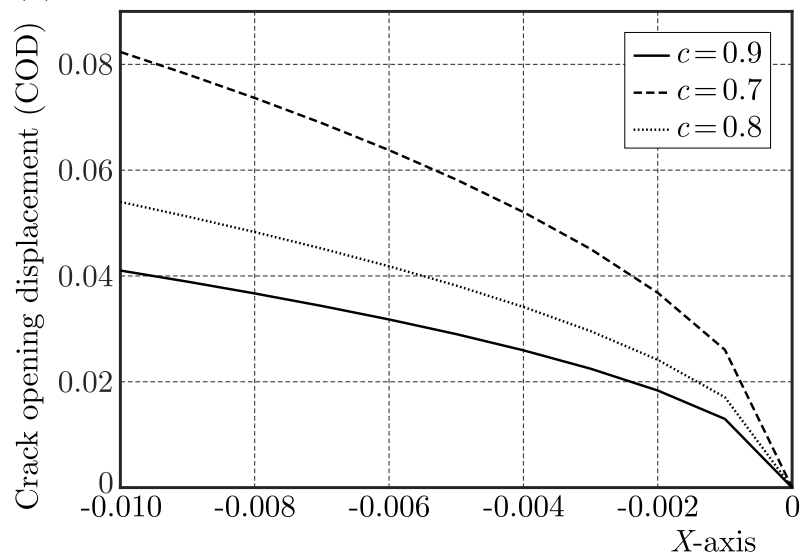

Fig. 6. COD vs. distance $x$ for Type- 2 material, (a) $c=0.2$ and (b) $h=4$

\section{Conclusion}

The problem of isotropic media with moving semi-infinite cracks has been solved. The boundary value problem is transformed to the standard Wiener-Hopf equation by applying Fourier transformation to get expressions of the Stress Intensity Factor and Crack Opening Displacement in 
(a)

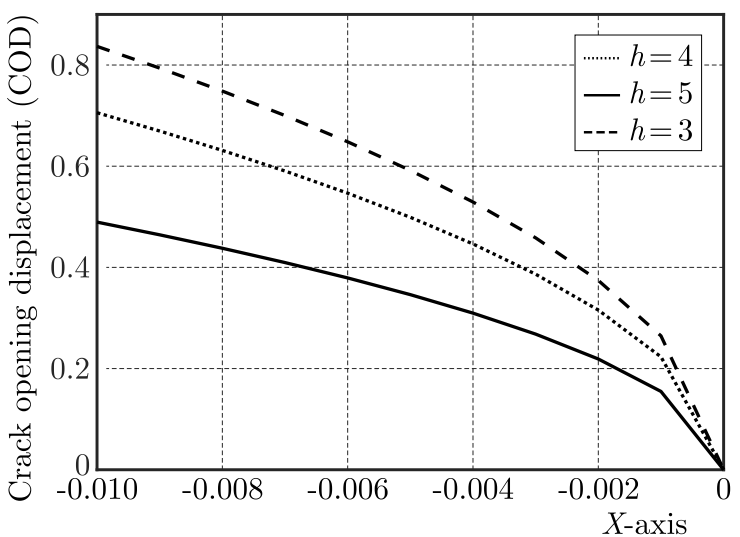

(b)

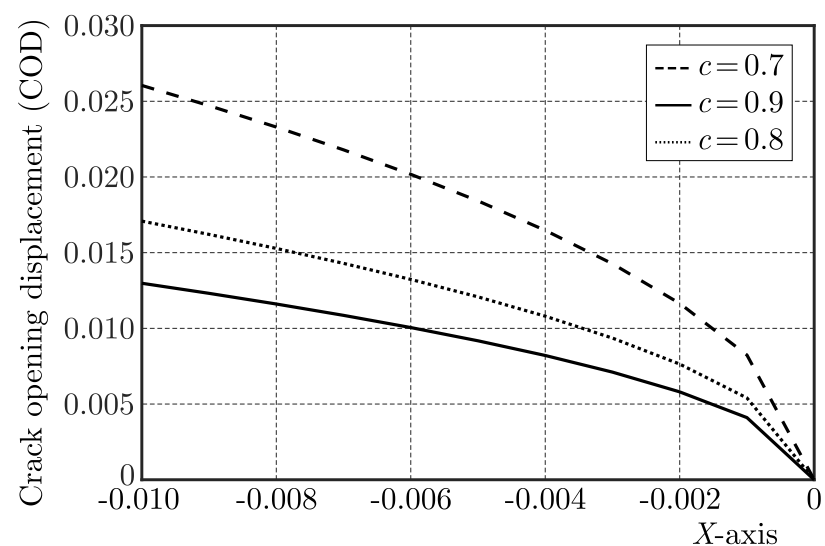

Fig. 7. COD vs. distance $x$ for Type-3 material, (a) $c=0.2$ and (b) $h=4$

asymptotic cases. The figures show the effects of different parameters on these SIF and COD. Firstly, the SIF value obtained from the expression and represented graphically shows variation of some parameters (such as crack depth $h$ from the surface, crack velocity $v$ ). The conclusion is that the SIF can be controlled and detained within a specific range. We can vary the same parameters in the case of the COD. To find the SIF and COD, the material properties also play the vital role. We have used three combinations of isotropic materials to obtain the above SIF and COD graphs. These results can also be applied to other isotropic materials that are very useful in engineering mathematics.

\section{Acknowledgement}

I would like to thank anonymous reviewers for their suggestion to improve the manuscript. This research work is financially supported by the University Grants Commission, India, Award No. 52047.

\section{References}

1. AtKinson C., List R.D., 1978, Steady state crack propagation into media with spatially varying elastic properties, International Journal of Engineering Science, 16, 717-730

2. Atkinson C., Popelar C.H., 1979, Antiplane dynamic crack propagation in a viscoelastic strip, Journal of Mechanics and Physics of Solids, 27, 5, 431-439

3. Bagheri R., Ayatollahi M., Mousavi S.M., 2016, Analytical solution of multiple moving cracks in functionally graded piezoelctric strip, Journal of Applied Mathematics and Mechanics, 36, 6, 777-792

4. Das A.N., Ghosh M.L., 1992, Two coplanar Griffith cracks moving along the interface of two dissimilar elastic medium, Engineering Fracture Mechanics, 41, 59-69

5. Georgiadis H.G., 1986, Complex variable and integral transform methods for elastodynamic solutions of cracked orthotropic strips, Engineering Fracture Mechanics, 24, 5, 727-735

6. Georgiadis H.G., Papadopoulos G.A., 1988, Cracked orthotropic strip with clamped boundaries, Journal of Applied Mathematics and Physics, 39, 573-578

7. Knauss W.H., 1966, Stresses in an infinite strip containing a semi-finite crack, Journal of Applied Mechanics, 33, 2, 356-362

8. Kuo M., 1998, Stress intensity factors for a semi-infinite plane crack under a pair of point forces on the faces, Journal of Elasticity, 30, 3, 197-209 
9. Kuo M., Chen T.Y., 1992, The Wiener-Hopf technique in elastodynamic crack problems with characteristic lengths in loading, Engineering Fracture Mechanics, 42, 5, 805-813

10. LI X.F., 2001, Closed-form solution for a mode-III interface crack between two bonded dissimilar elastic layers, International Journal of Fracture, 109, L3-L8

11. Lowengrub M., 1975, A pair of coplanar cracks at the interface of two bonded dissimilar elastic half-planes, International Journal of Engineering Science, 13, 731-741

12. Lowengrub M., Srivastava K.N., 1968, On two coplanar Griffith cracks in an infinite elastic medium, International Journal of Engineering Science, 6, 359-362

13. Mandal P., Mandal S.C., 2017, Interface crack at orthotropic media, International Journal of Applied and Computational Mathematics, 3, 4, 3253-3262

14. Matczyński M., 1973, Motion of a crack in antiplane strain of an elastic strip, Archive of Mechanics, 25, 823

15. Nandi A., Mandal S.C., 2017, Diffraction of sh-waves with cracks in composite media, Mechanics of Advanced Materials and Structures, 25, 11, 881-888

16. Nilsson F., 1972, Dynamic stress intensity factors for finite strip problems, International Journal of Fracture Mechanics, 8, 4, 403-411

17. Nilsson F., 1973, Erratum to dynamic stress intensity factors for finite strip problems, International Journal of Fracture, 9, 4, 477

18. Noble B., 1958, Method Based on the Wiener-Hopf Technique, Pergamon Press, New York

19. Nourazar M., Ayatollahi M., 2016, Multiple moving interfacial cracks between two dissimilar piezoelectric layers under electromechanical loading, Smart Materials and Structures, 25, 7

20. Rice J.R., 1967, Discussion: stresses in an infinite strip containing a semi-infinite crack, Journal of Applied Mechanics, 34, 1, 248-249

21. Sarkar J., Ghosh M.L., Mandal S.C., 1991, Scattering of antiplane shear wave by a propagating crack at the interface of two dissimilar elastic media, Proceedings of the Indian Academy of Mathematical Sciences, 101, 183-194

22. SiH G.C., 1968, Some elastodynamic problems of cracks, International Journal of Fracture Mechanics, 1, 51-68

23. Srivastava K.N., Palaiya R.M., Karaulia D.S., 1980, Interaction of antiplane shear waves by a Griffith crack at the interface of two bonded dissimilar elastic half spaces, International Journal of Fracture, 16, 349-358

24. Wang C.Y., Rubio-Gonzalez C., Mason J.J., 2001, The dynamics stress intensity factor for a semi-infinite crack in orthotropic materials with concentrated shear impact loads, International Journal of Solids and Structure, 38, 1265-1280

25. Wu X.F., Dzenis Y.A., FAn T.Y., 2003, Two semi-infinite interfacial cracks between two bonded dissimilar elastic strips, International Journal of Engineering Science, 41, 15, 1699-1710

26. Wu X.F., Lilla E., Zou W.S., 2002, A semi-infinite interfacial crack between two bonded dissimilar elastic strips, Archive of Applied Mechanics, 72, 630-636

27. Yoffe E.H., 1951, The moving Griffith crack, Philosophical Magazine, 42, 739-750 